\title{
Does guar gum improve post-prandial hyperglycaemia in humans by reducing small intestinal contact area?
}

\author{
BY N. A. BLACKBURN,* A. M. HOLGATE AND N. W. READ† \\ Clinical Research Unit, Royal Hallamshire Hospital, Sheffield S10 $2 J F$
}

(Received 27 October 1983 - Accepted 21 March 1984)

\begin{abstract}
1. The possibility that viscous polysaccharides, such as guar gum, could lower post-prandial blood glucose levels in part by restricting carbohydrate solutions to a smaller area of small intestine was investigated in twenty healthy human volunteers.

2. Addition of guar gum $(22.5 \mathrm{~g} / \mathrm{l})$ delayed the mouth-to-caecum transit time of a hypotonic lactulose drink, but did not affect gastric emptying.

3. When a $250 \mathrm{ml}$ solution containing 50 g glucose was confined to a $550 \mathrm{~mm}$ length of intestine by an occluding balloon attached to an intestinal tube, maximum blood glucose response was significantly reduced $(\mathrm{P}<0.05)$ though only by $0.9 \mathrm{mmol} / 1$.

4. Addition of guar gum $(36 \mathrm{~g} / \mathrm{l})$ had no effect on the distribution of a radio-labelled glucose drink (250 ml; $200 \mathrm{~g}$ glucose $/ 1$ ) in the small intestine, monitored using a gamma camera, although it significantly delayed gastric emptying ( $\mathrm{t}_{1}$ (min): guar gum v. control 115 (SE 15) v. 73 (SE 8)).

5. Reduced contact area is unlikely to be one of the mechanisms by which guar gum improves glucose tolerance.
\end{abstract}

It is now well established that the addition of guar gum (or other viscous polysaccharides) to carbohydrate-rich test meals or oral glucose loads lowers post-prandial hyperglycaemia in normal and diabetic humans (Jenkins et al. 1976, 1977, 1978; Gassull et al. 1976; Morgan et al. 1979; Wahlqvist, 1979). This is thought to be caused by a reduction in the rate of glucose absorption due in part to a delay in the delivery of carbohydrate to the small intestine (Holt et al. 1979, 1981) and in part to an inhibition of convection within the intestinal lumen, preventing access of glucose to the intestinal epithelium (Flourie et al. 1982; Blackburn et al. 1984). However, the observations that ingestion of viscous polysaccharides can also slow small-bowel transit time (Bueno et al. 1981; Forman \& Schneeman, 1982) suggests that guar gum might also reduce glucose absorption by reducing the spread of luminal contents along the small intestine and limiting the area of mucosa (and hence the number of transport sites) in contact with nutrients. We have carried out a series of studies in normal volunteers to test whether this mechanism might contribute towards the hypoglycaemic action of guar gum.

\section{METHODS}

\section{Subjects}

Studies were performed on a total of twenty healthy volunteers (fifteen male, five female) aged between 20 and 27 years, all of whom had given their written, informed consent. Female subjects were only studied if they were using a reliable form of contraception, if they were in the first $10 \mathrm{~d}$ of their menstrual cycle, and if they signed a form stating that they were not pregnant and were unlikely to become pregnant during the course of the study. All experimental protocols were approved by the Ethical Subcommittee of the Sheffield Area Health Authority (Teaching), Southern District, in April 1981.

In studies using radioactive markers, the whole-body radiation dose from $100 \mu \mathrm{Ci}$

* Present address: Department of Pharmacology, Reckitt and Coleman Pharmaceuticals, Dansom Lane, Hull HU8 7DS.

$\dagger$ For reprints. 
[ ${ }^{90 \mathrm{~m}}$ Technecium] sulphur colloid was less than 2 mrads, and the total dose to the gonads was less than 10 mrads.

\section{Measurement of gastric emptying and small intestinal transit time}

Paired studies were performed in six subjects (four male, two female) to determine the effect of guar gum on the gastric emptying and small-bowel transit time (SBTT) of a $400 \mathrm{ml}$ drink containing $10 \mathrm{~g}$ lactulose (osmolality $70 \mathrm{mosm} / \mathrm{l}$ ) and $50 \mu \mathrm{Ci}\left[{ }^{99 \mathrm{~m}} \mathrm{Tc}\right.$ ] S colloid, with or

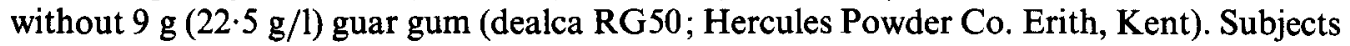
fasted overnight before each test. Then, after ingesting the drink, they lay supine for the remainder of the study. The rate of gastric emptying was determined by monitoring the decline in radioactivity over the fundus of the stomach using a single-crystal scintillation detector (Read et al. 1980). SBTT was determined by measuring the concentration of hydrogen in serial samples of end expiratory air collected every $10 \mathrm{~min}$ throughout the study, and was taken as the time from ingesting the drink to the first sustained rise in breath $\mathbf{H}_{2}$ concentration (Read et al. 1980). The latter was defined as a rise of at least $3 \mu 1 / 1$ above basal values sustained for at least three consecutive 10 min readings. The order in which the experiments were carried out was randomized.

\section{Effect of restricting contact area on glucose tolerance}

Paired studies were carried out in eight subjects (seven male, one female) to determine whether restricting the spread of a glucose solution in the small intestine had any effect on the blood glucose response to a $50 \mathrm{~g}$ glucose load. After an overnight fast, the subjects swallowed a flexible two-lumen probe, which consisted of two polyvinyl tubes bonded together and weighted at the distal end with a mercury-filled double balloon. The probe was positioned using fluoroscopy so that the infusion port, which was $450 \mathrm{~mm}$ proximal to the balloon, was in the mid-duodenum. A solution of $50 \mathrm{~g}$ glucose in $250 \mathrm{ml}$ water was then infused through this port into the duodenum at a rate of $5 \mathrm{ml} / \mathrm{min}$. In control studies, the $\mathrm{Hg}$ was aspirated so that the balloon presented as little obstruction to flow as possible. In test studies, the balloon was inflated with approximately $25 \mathrm{ml}$ of air so as to occlude the small intestinal lumen, confining the infused glucose solution to approximately $550 \mathrm{~mm}$ of small intestine. Blood samples were withdrawn from a butterfly needle, situated in a forearm vein, $10 \mathrm{~min}$ before and immediately before the infusion started, and thereafter at $20 \mathrm{~min}$ intervals for $2 \mathrm{~h}$. The blood samples were analysed for glucose (glucose oxidase (EC 1.1.3.4) method) and insulin (radioimmunoassay). The order of the studies was randomized.

\section{Measurement of distribution of a glucose drink}

A gamma camera linked to a dedicated mini-computer was used to determine the effects of guar on the distribution of a radio-labelled drink throughout the stomach and small intestine (Read et al. 1983). Paired studies were carried out in six subjects (four male, two female). After an overnight fast, the subjects ingested a glucose drink $(50 \mathrm{~g}$ glucose, $200 \mathrm{ml}$ water, $50 \mathrm{ml}$ diabetic orange squash) labelled with $100 \mu \mathrm{Ci}$ [ $\left.{ }^{89 \mathrm{~m}} \mathrm{Tc}\right] \mathrm{S}$ colloid, with or without $9 \mathrm{~g}$ guar gum $(36 \mathrm{~g} / \mathrm{l})$. They then lay supine on an air mattress and the gamma camera (model $400 \mathrm{~T}$; Elscint, Israel) was positioned over the abdomen at a distance of approximately $100 \mathrm{~mm}$. Cumulative images of the distribution of the radio-label within the abdominal cavity were recorded every $5 \mathrm{~min}$ for $2 \mathrm{~h}$ and stored on a data-processing disc for future analysis. Venous blood samples for glucose analysis were withdrawn from an indwelling butterfly needle $10 \mathrm{~min}$ before and immediately before the drink was ingested, and thereafter at $10 \mathrm{~min}$ intervals for $2 \mathrm{~h}$. The order of the guar gum and control studies was randomized. 


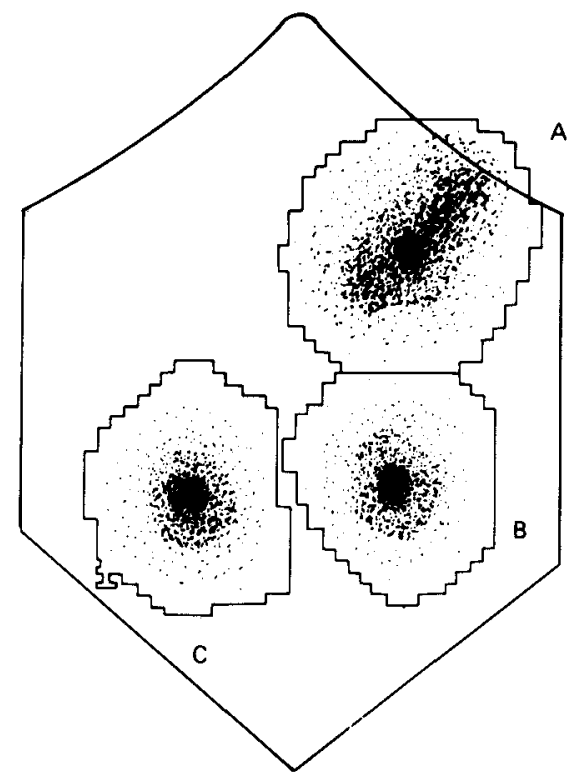

Fig. 1. An illustration of the three regions of interest that were outlined on the gamma camera images of human subjects. A, stomach; B and C, two areas of the upper small intestine.

\section{Processing of gamma camera data}

After the radio-label left the stomach, it always accumulated in two discrete areas in the abdomen in every subject tested, whether or not they had ingested guar gum; one below the stomach and to the left of the midline (B), the other towards the right of the midline (C) (Fig.1). A totalized image of the whole study was displayed and regions of interest were then outlined around the stomach (region A) and the two areas in the abdomen (Fig.1). The radioactivity within these regions (corrected for decay) was plotted every 5 min throughout each study to yield profiles for gastric emptying and filling of regions B and $\mathrm{C}$.

\section{Statistical analysis}

Paired Student's $t$ tests were used to assess the statistical significance of the results.

\section{RESULTS}

Gastric emptying and small bowel transit time

The addition of $9 \mathrm{~g}$ guar gum to a hypotonic drink containing lactulose significantly delayed SBTT (control 152 (SE 20) min, guar gum 249 (SE 32) min; $P<0.01$ ) but did not significantly alter values for the half-time $\left(t_{2}\right)$ for gastric emptying (control 23 (SE 7) min, guar gum 25 (SE 6) $\min ; P>0 \cdot 1$ ).

\section{Effect of contact area on glucose tolerance}

Confining the infused glucose solution to a $550 \mathrm{~mm}$ segment of small intestine using an occluding balloon reduced the blood glucose concentrations at $40 \mathrm{~min}(P<0.02)$ and $60 \mathrm{~min}(P<0.05)$ after the start of the glucose infusion (Fig.2), although the average reduction in the maximum glucose response was only $0.9 \mathrm{mmol} / 1$. Plasma insulin levels were not significantly lower $(P>0 \cdot 1)$ when the glucose solution was confined (Fig.2). 


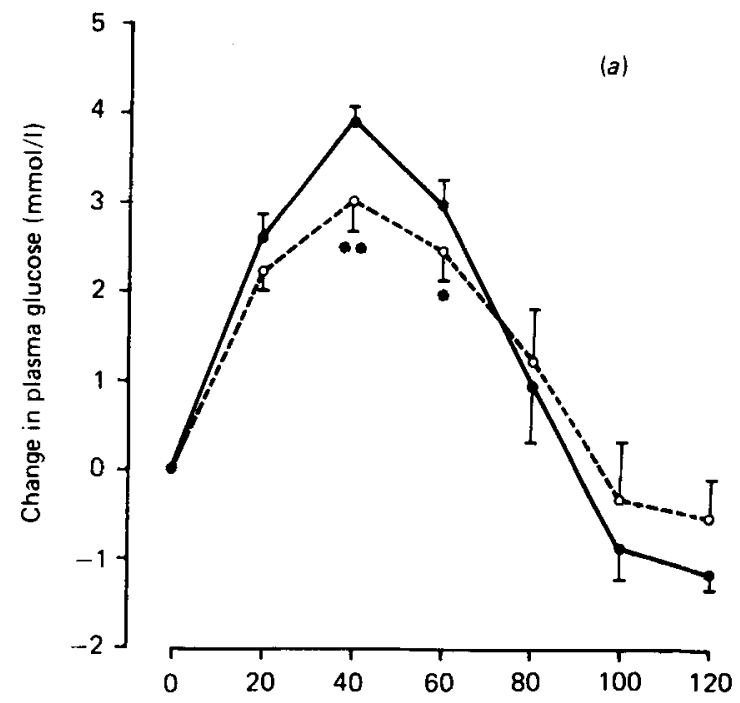

(b)

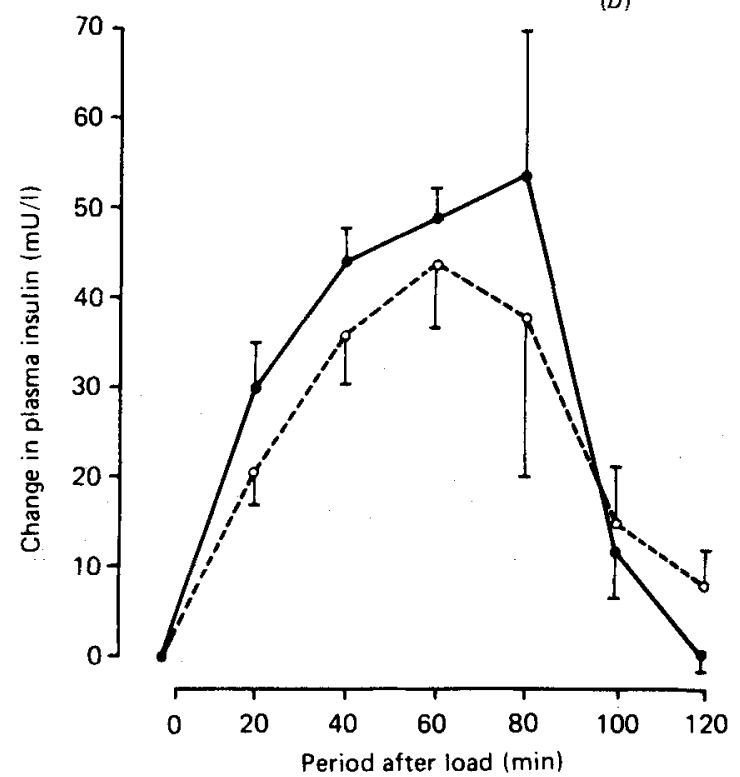

Fig. 2. ( $a$ ) Blood glucose and $(b)$ plasma insulin levels following infusion of $50 \mathrm{~g}$ glucose in $250 \mathrm{ml}$ liquid directly into the duodenum of human subjects. The glucose load was either confined to a $550 \mathrm{~mm}$ segment of intestine using an occluding balloon $(O)$ or allowed to flow normally along the small intestine $(O)$. Results are expressed as means, with their standard errors, of eight observations. ${ }^{*} P<0.05$, ** $P<0.02$.

\section{Measurement of distribution of a glucose drink}

Glucose tolerance was significantly improved by the addition of $9 \mathrm{~g}$ guar gum to the drink containing glucose. Blood glucose levels were significantly lower 30 and $40 \min (P<0.05)$ after the drink was ingested (Fig.3). The addition of guar gum also delayed gastric emptying of the glucose drink ( $t_{1}(\mathrm{~min})$ : control, 73 (SE 8), guar gum 115 (SE 15) $P<0.01$ ) and hence the rate of entry of radio-label into the small intestinal regions B and C (Fig. 4). However, 


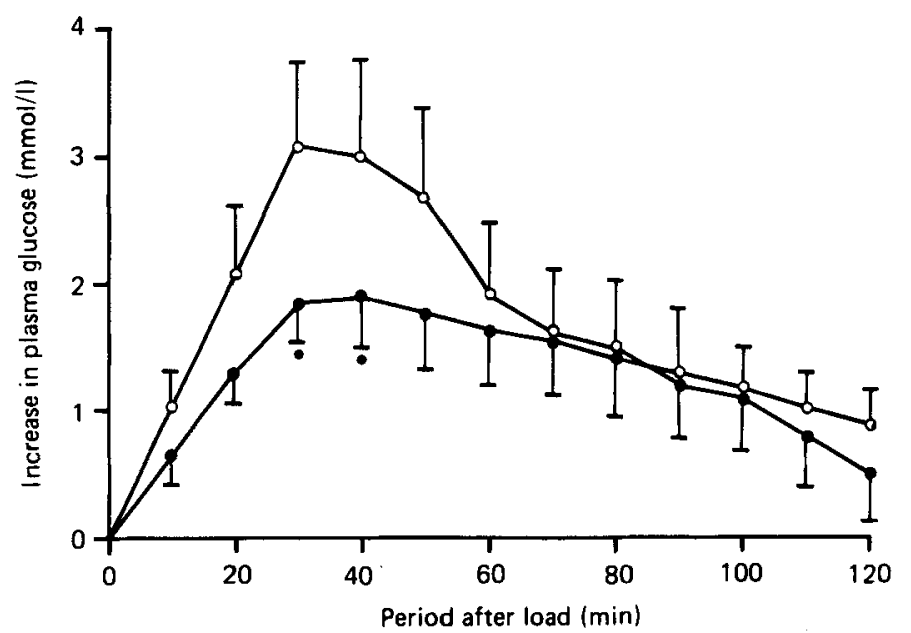

Fig. 3. Blood glucose levels of human subjects following ingestion of $50 \mathrm{~g}$ glucose in a $250 \mathrm{ml}$ orange drink, with (O) or without $(O) 9 \mathrm{~g}$ guar gum. Results are expressed as means, with their standard errors, of six observations. ${ }^{*} P<0.05$.

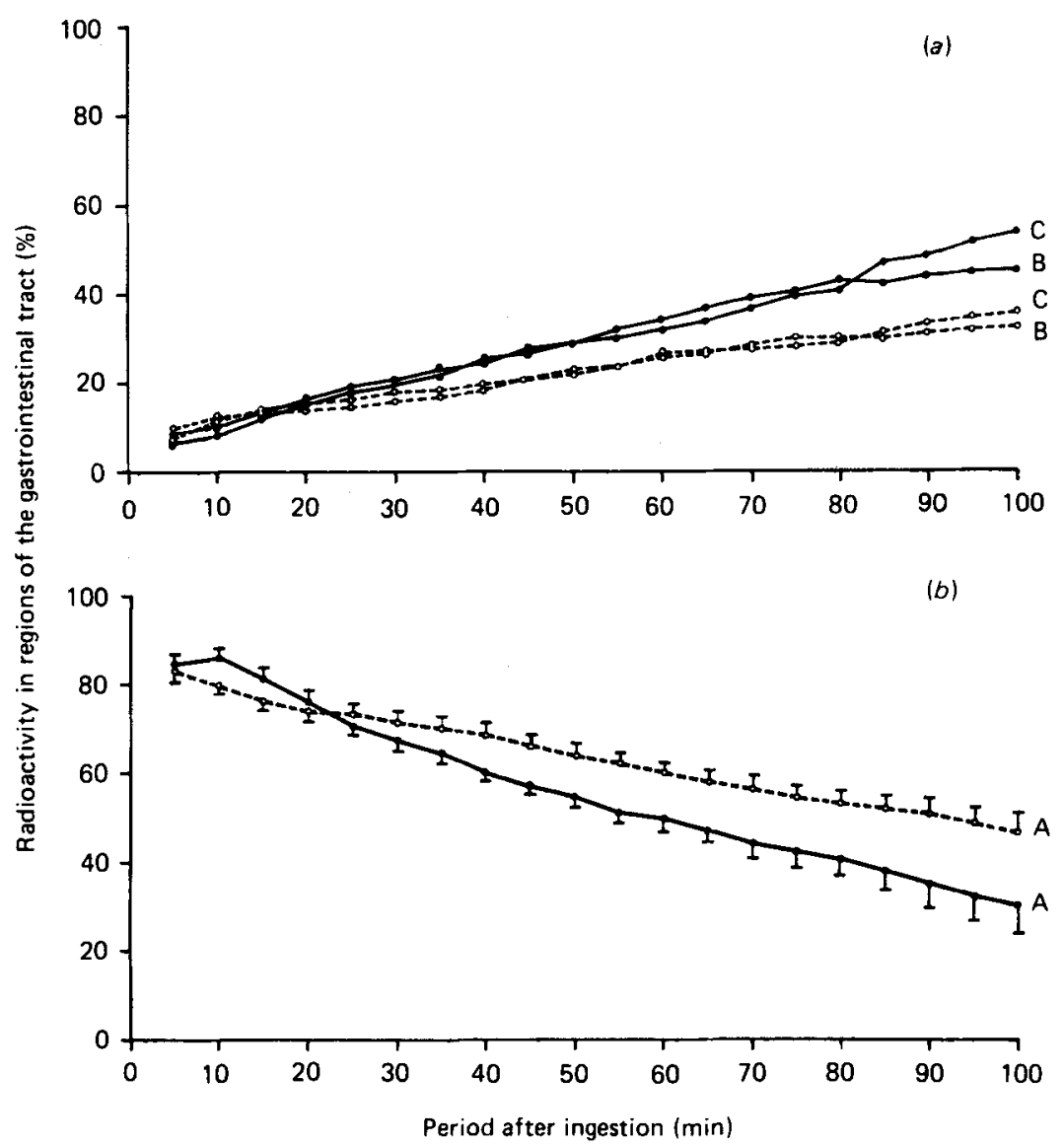

Fig. 4. Curves for $(a)$ filling of small intestine and $(b)$ gastric emptying following ingestion by human subjects of $50 \mathrm{~g}$ glucose in a $250 \mathrm{ml}$ orange drink, with $(O)$ or without (O) $9 \mathrm{~g}$ guar gum. Results are expressed as means, with their standard errors, of six observations of the percentage of the total counts in regions A, B and C throughout the study (see Fig. 1). For clarity, error bars are omitted in (a). 

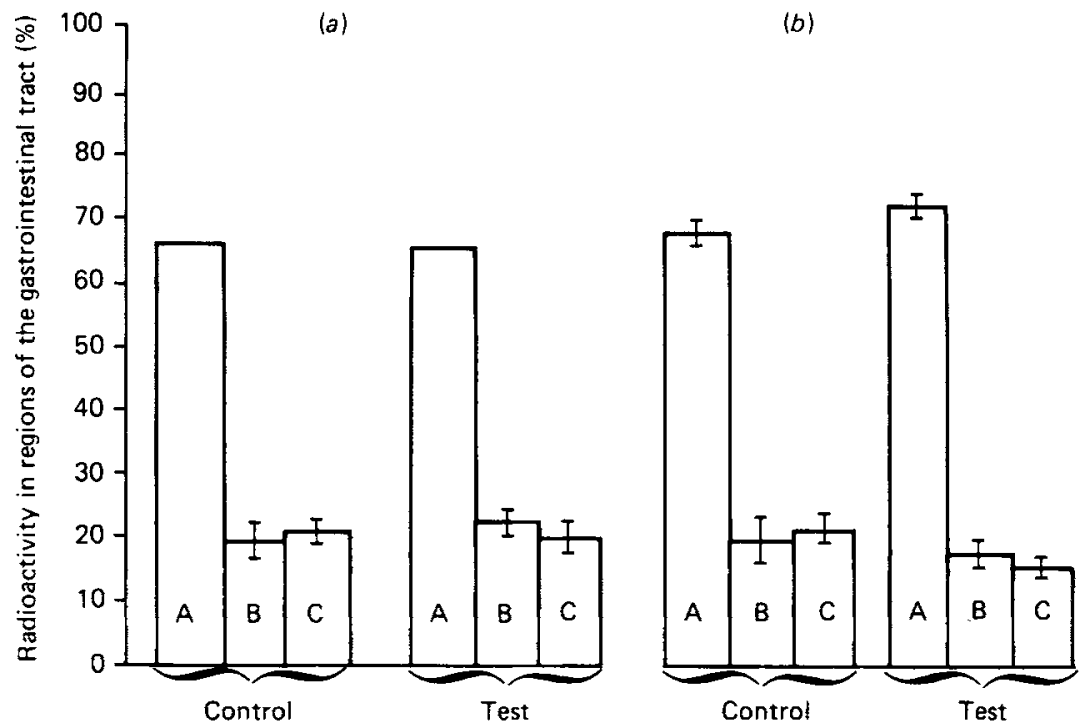

Fig. 5. Distribution of radio-label (expressed as a percentage of the total counts in regions A, B and $\mathrm{C}$ (see Fig. 1) at the end of the first 5 min counting period) in regions $\mathrm{A} ; \mathrm{B}$ and $\mathrm{C}$ at the time when (a) $67 \%$ of radio-label remained in the stomach or $(b)$ at the time when peak blood glucose levels occurred $(t 30 \mathrm{~min})$. Values are means, with their standard errors, of six observations. The fact that the total counts in $\mathrm{A}+\mathrm{B}+\mathrm{C}$ exceeded $100 \%$ at the later stages of the study can be explained by the greater proportion of isotope in the small intestine and hence nearer the camera head (Jacobs et al. 1982).

when individual profiles were examined either at the time when the peak glucose response occurred $(t 30 \mathrm{~min})$, or at the time when the same amount $(67 \%)$ of radioactivity remained in the stomach, there were no significant differences in the relative distribution of the radiolabel in the two regions of small intestine between control and test studies (Fig.5).

\section{DISCUSSION}

Our initial experiments showed that when guar gum was added to a drink containing an unabsorbable sugar marker, the rate of passage of that drink through the small intestine was retarded although gastric emptying was unaffected. This result supported previous evidence showing that guar gum delays small-bowel transit in animals and, more recently, in man (Bueno et al. 1981; Forman \& Schneeman 1982), and raised the possibility that guar gum may reduce post-prandial hyperglycaemia in part by limiting the distribution of a meal or glucose load down the length of the intestine so that the nutrients were exposed to fewer absorbing sites. The observation that gastric emptying of the lactulose solution was unaffected by guar gum, whereas the emptying of glucose solutions was delayed suggests that the effect of guar gum becomes obvious only when gastric emptying is already slowed, for example, by the hypertonicity of the glucose solution. This result is compatible with information from Hunt (1954), who could not demonstrate any effect of viscosity on the emptying of a hypotonic sucrose drink in man, whereas other workers have shown that the addition of viscous polysaccharides slows the emptying of hypertonic glucose solutions in man (Holt et al. 1979).

Experiments in which a glucose solution was confined to only $550 \mathrm{~mm}$ of small intestine by inflating an occluding balloon, confirmed that reductions in contact area could reduce the blood glucose response to a standard glucose load, although the reduction was only 
small (approximately $23 \%$ ). It is possible that a greater reduction in blood glucose concentrations could have been achieved by infusing at a more rapid rate, although the rate was chosen to be similar to the average rate of emptying of a similar glucose solution from the stomach (Blackburn et al. 1984).

Having shown that a reduction in contact area could theoretically play a role in limiting post-prandial hyperglycaemia, we used a gamma camera to try to establish whether guar gum affected the way in which a glucose drink was distributed throughout the small intestine. Analysis of these results shows that after leaving the stomach the glucose drink appeared to accumulate in two areas (B and C, Fig.1). Guar gum did not appear to affect the distribution of counts in regions $\mathrm{B}$ and $\mathrm{C}$ either at the time when the maximum blood glucose concentration occurred ( $t 30 \mathrm{~min}$ ), or at the time when the same amount of radio-label $(67 \%)$ remained in the stomach. Thus, these results do not support the hypothesis that guar gum can reduce post-prandial hyperglycaemia by limiting contact area.

It seems likely that areas B and C were both in the jejunum, since they both occupied the upper and middle abdomen and since the isotope started to accumulate in both areas very soon after ingestion. While no counts appeared to accumulate in the colon, we cannot exclude the possibility that some radioactive counts were in the ileum (in the pelvis and outside of the field of view of the gamma camera) during the later stages of the study. However, this factor is unlikely to be of relevance since the total abdominal counts in the guar gum studies, normalized to the counts present shortly after ingestion of the drink, were not significantly different from the normalized abdominal counts in control studies at equivalent times after ingestion. In other words, there was no evidence that different amounts of isotope were leaving the field of view (middle and upper abdomen) in the guar gum study compared with the control study. Moreover, as we did not observe a large effect on post-prandial blood glucose levels when the glucose solution was confined to only $550 \mathrm{~mm}$ of small intestine, it is unlikely that the entry of glucose into the ileum could exert a measurable effect of glucose tolerance unless some other factor, such as the release of a hormone, for example, were involved.

In conclusion, our studies have indicated that reduction in contact area is unlikely to be one of the mechanisms by which guar gum improves glucose tolerance in normal subjects under the acute conditions of these experiments and that other effects, such as reduction in convection within the intestinal lumen and delayed gastric emptying, are more important. These experiments, however, do not necessarily exclude the possibility that a reduction in contact area may be important in the improvement in post prandial hyperglycaemia, seen in diabetics after chronic administration of viscous polysaccharides.

\section{REFERENCES}

Blackburn, N. A., Redfern, J. S., Jarjis, H., Holgate, A. M., Hanning, I., Scarpello, J. H. B., Johnson, I. T. \& Read, N. W. (1984). Clinical Science 66, 329-336.

Bueno, L., Praddaude, F., Fioramonti, J. \& Ruckebusch, Y. (1981). Gastroenterology 80, 701-707.

Flourie, B., Vidon, N., Florent, C. \& Bernier, J. J. (1982). Gut 23, A911.

Forman, L. P. \& Schneeman, B. O. (1982). Journal of Nutrition 112, 528-533.

Gassull, M. A., Goff, D. V., Haisman, P., Hockaday, T. D. R., Jenkins, D. J. A., Jones, K., Leeds, A. R. \& Wolever, T. M. S. (1976). Journal of Physiology, London 259, 52-53.

Holt, S., Heading, R. C., Carter, D. C., Prescott, L. F. \& Tothill, P. (1979). Lancet i, 636-639.

Holt, S., Heading, R. C. \& Clements, J. (1981). Gastroenterology 80, 1611-1612.

Hunt, J. N. (1954). Lancet i, 17-18.

Jacobs, F., Akkermans, L. M. A., Oei Hong Yoe, Hoekstra, A. \& Wittebol, P. (1982). Motility of the Digestive Tract, pp. 233-240 [M. Wienbeck, editor]. New York: Raven Press.

Jenkins, D. J. A., Leeds, A. R., Gassull, M. A., Cochet, B. \& Alberti, K. G. M. M. (1977). Annals of International Medicine 86, 20-23.

Jenkins, D. J. A., Leeds, A. R., Wolever, T. M. S., Goff, D. V., Alberti, K. G. M. M., Gassull, M. A. \& Hockaday, T. D. R. (1976). Lancet ii, 172-174. 
Jenkins, D. J. A., Wolever, T. M. S., Leeds, A. R., Gassull, M. A., Haisman, P., Dilawari, J., Goff, D.V., Metz, G. L. \& Alberti, K. G. M. M. (1978). British Medical Journal i, 1392-1394.

Morgan, L. M., Goulder, T. J., Tsiolakis, D., Marks, V. \& Alberti, K. G. M. M. (1979). Diabetologia 17, 85-89.

Read, N. W., Al-Janabi, M. N., Bates, T. E., Barber, D. C. (1983). Gastroenterology 84, 1568-1572.

Read, N. W., Miles, C. A., Fisher, D., Holgate, A. M., Kime, N. D., Mitchell, M. A., Reeve, A. M., Roche, T. B. \& Walker, M. (1980). Gastroenterology 79, 1276-1282.

Wahlqvist, M. L., Morris, M. J., Littlejohn, G. O., Bond, A. \& Jackson, R. V. (1979). Australian and New Zealand Journal of Medicine 9, 154-158. 